\title{
Temperature-Dependent Refractive Index of Quartz at Terahertz Frequencies
}

\author{
Christopher L. Davies $^{1}$ • Jay B. Patel ${ }^{1}$. Chelsea Q. Xia ${ }^{1}$ - Laura M. Herz ${ }^{1}$. \\ Michael B. Johnston ${ }^{1}$
}

Received: 2 July 2018 / Accepted: 30 August 2018 / Published online: 13 September 2018

(C) The Author(s) 2018

\begin{abstract}
Characterisation of materials often requires the use of a substrate to support the sample being investigated. For optical characterisation at terahertz frequencies, quartz is commonly used owing to its high transmission and low absorption at these frequencies. Knowledge of the complex refractive index of quartz is required for analysis of time-domain terahertz spectroscopy and optical pump terahertz probe spectroscopy for samples on a quartz substrate. Here, we present the refractive index and extinction coefficient for $\alpha$-quartz between $0.5 \mathrm{THz}$ and $5.5 \mathrm{THz}\left(17-183 \mathrm{~cm}^{-1}\right)$ taken at $10,40,80,120,160,200$ and $300 \mathrm{~K}$. Quartz shows excellent transmission and is thus an ideal optical substrate over the $\mathrm{THz}$ band, apart from the region $3.9 \pm 0.1 \mathrm{THz}$ owing to a spectral feature originating from the lowest energy optical phonon modes. We also present the experimentally measured polariton dispersion of $\alpha$-quartz over this frequency range.
\end{abstract}

Keywords Quartz $\cdot$ Silica $\cdot \mathrm{SiO}_{2} \cdot$ Refractive index $\cdot$ Polariton · Dispersion · Terahertz · Time-domain spectroscopy · Optical pump terahertz probe OPTPS

\section{Introduction}

Quartz has been extensively utilised as a substrate for optical characterisation of non-free standing samples, both in the near-infrared to visible region [1-4], and the terahertz region [5-10] of the electromagnetic spectrum, owing to quartz having low

Michael B. Johnston

michael.johnston@physics.ox.ac.uk

1 Department of Physics, University of Oxford, Clarendon Laboratory, Parks Road, Oxford OX1 3PU, UK 
absorption and high transmission in these regions [11, 12]. Samples supported by a substrate form a heterostructure and optical characterisation of these samples will often require optical modelling of the sample-substrate heterostructure [10,13]. For precise optical modelling of heterostructures with quartz, knowledge of the complex refractive index, $\tilde{n}=n+i \kappa$, of quartz is required where $n$ is the refractive index and $\kappa$ is the extinction coefficient. While there have been reports previously on the complex refractive index of quartz at room temperature and low temperature [12], little is known on the temperature-dependence of the complex refractive index of quartz in the terahertz region.

Quartz is an earth-abundant mineral form of tetrahedrally bonded silica (i.e. silicon dioxide, $\mathrm{SiO}_{2}$ ). At temperatures below $846 \mathrm{~K}$, the stable crystalline form of quartz is the $\alpha$-phase which is uniaxial and has a chiral structure that may be right or left handed [14]. Thus light propagating along the optic axis (also called $c$-axis or $z$ axis) suffers no birefringence but experiences optical activity, while light propagating along other directions exhibits birefringence. At terahertz frequencies, $x$-cut quartz has been shown to be highly birefringent over the range $100 \mathrm{GHz}$ to $3.5 \mathrm{THz}$ [15], and stacks of birefringent quartz plates have been used to create an achromatic quarter waveplate operating at terahertz frequencies [16]. When used as substrates for optical and $\mathrm{THz}$ spectroscopy, wafers of $z$-cut quartz are typically used in order to avoid birefringence affecting light propagating normal to the surface of the wafer, which is the usual measurement geometry.

$\alpha$-quartz has a 3-formula unit (i.e. 9 atom) basis in its trigonal primitive unit cell (space group $P 3_{2} 21$ ) and thus possesses 24 optical and 3 acoustic phonon branches. The lowest energy pair of optical phonon modes is both infrared and Raman active. These modes have been calculated to have an energy of $133.3 \mathrm{~cm}^{-1}$ for the TO mode and $133.4 \mathrm{~cm}^{-1}$ for the LO mode at the Brillouin zone centre assuming a temperature of $0 \mathrm{~K}$ [17]. The small splitting of the energy degeneracy of these $E$ symmetry TO and LO modes is expected as a consequence of anharmonicity, but is difficult to observe experimentally owing to phonon-lifetime broadening exceeding the splitting [18]. The next highest infrared-active mode is also expected to be a (near) doubly degenerate $E$-mode at $262 \mathrm{~cm}^{-1}[17,19]$. Bréhat and Wyncke used infrared spectroscopy to measure phonon-modes at $128 \mathrm{~cm}^{-1}(\sim 3.8 \mathrm{THz})$ and $265 \mathrm{~cm}^{-1}(\sim 8 \mathrm{THz})$ in close agreement with theory [12]. There is also an $A_{1^{-}}$ symmetry phonon at an energy of $\sim 220 \mathrm{~cm}^{-1}[17,19]$; however, this mode is not infrared active. Since the lowest energy $E$-mode $\left(128 \mathrm{~cm}^{-1}\right)$ lies close to the region generally of interest in terahertz time-domain spectroscopy (TDS) and optical pump terahertz probe spectroscopy (OPTPS) measurements, it is important to understand the temperature-dependence of this phonon mode and how it could affect TDS and OPTPS measurements.

Previous measurements of the complex refractive index at terahertz frequencies were performed using either Fourier spectroscopy $[20,21]$ or infrared grating spectroscopy [22]. Interestingly, one of the earliest demonstrations of THz-TDS was by Grischkowsky et al. [23] who extracted the refractive index and absorption coefficient of quartz at room temperature over a range from $0.2-2.0 \mathrm{THz}$. Over the past 
decade the bandwidth available to terahertz TDS and OPTPS has increased owing to improvements of emitters and detectors [24]. These improvements now allow the complex refractive index of materials to be measured with high precision over a large frequency range.

Here, we measure the complex refractive index of $\alpha$-quartz $\left(\mathrm{SiO}_{2}\right)$ at 10,40 , $80,120,160,200$ and $300 \mathrm{~K}$, between $0.5 \mathrm{THz}$ and $5.5 \mathrm{THz}$ using terahertz TDS. The electric field of the terahertz probe was aligned perpendicular to the $c$-axis of the $\alpha$-quartz sample. We present the temperature-dependence of the $\sim 3.9 \mathrm{THz}$ phonon mode ( $E$-symmetry mode [25]), showing that the phonon mode red shifts with increasing temperature from $3.96 \mathrm{THz}$ at $10 \mathrm{~K}$ to $3.86 \mathrm{THz}$ at $300 \mathrm{~K}$. The oscillator strength of the $3.9 \mathrm{THz}$ phonon mode also decreases with increasing temperature. We also show that THz-TDS allows the dispersion relation for the upper and lower branches of quartz's phonon-polariton dispersion to be determined experimentally.

\section{Experimental Methods}

\subsection{Terahertz Time-domain Spectroscopy (TDS)}

A terahertz time-domain spectrometer built at Oxford was used for this study. The system has been described previously [26]. Briefly, the output of an amplified Ti:Sapphire laser system with $30 \mathrm{fs}$ pulses at a repetition rate of $5 \mathrm{kHz}$ with a central wavelength of $800 \mathrm{~nm}$ and an average power of $4 \mathrm{~W}$ was split into two beam paths: a probe beam; and a gate beam. Terahertz pulses were generated using a spintronic emitter [27, 28]. Terahertz pulses were detected by electro-optic sampling using a $0.2 \mathrm{~mm}$ thick $\mathrm{GaP}(110)$ crystal, Wollaston prism and a pair of balanced photodiodes. Temperature-dependent TDS measurements of quartz were performed using a cold-finger cryostat (Oxford Instruments). By measuring the signal in the presence and absence of the sample the terahertz transmission in the frequency domain was obtained by Fourier transforming the signal and dividing the sample signal by the reference.

\subsection{Fourier Transform Infrared Spectroscopy (FTIR)}

The reflection $(R)$ and transmission $(T)$ spectra were measured from 100 to $350 \mathrm{~cm}^{-1}$ using a Bruker Vertex 80v Fourier transform infrared (FTIR) spectrometer with a globar source. A liquid helium cooled silicon bolometer (Infrared Laboratories Inc.) and Mylar beamsplitter were used for the measurements. Reflection and transmission spectra were converted into extinction coefficient spectra, $\kappa$ by

$$
\kappa(\bar{v})=\frac{-1}{4 \pi \bar{v} d} \ln \left(\frac{T}{1-R}\right),
$$

where $d$ is the thickness of the sample and $\bar{v}$ is the wavenumber. 


\section{Results}

\subsection{Temperature-dependent Refractive Index of $\alpha$-Quartz}

Single-crystal $\alpha$-quartz wafers were purchased from Bright Crystals Technology Inc. The wafers were $z$-cut to an accuracy of $\pm 15^{\prime}$, had a specified thickness of $2 \pm 0.05 \mathrm{~mm}$ and diameter of approximately $13 \mathrm{~mm}$. The wafers were doublesided polished with a scratch/dig specification of 40/20. Time-domain spectroscopy, detailed in Section 2, was used to measure the amplitude and phase of the transmission through $\alpha$-quartz of a broadband terahertz pulse in the time-domain. The thickness of the particular wafer used in the data presented here was measured using a micrometer screw gauge at the centre and edge of the wafer and was determined to be $2.05 \pm 0.03 \mathrm{~mm}$. The custom-built TDS system at Oxford is well-suited for determining the dielectric function at different temperatures as the cryostat and $\mathrm{THz}$ optics are enclosed in the same vacuum space, avoiding the need for cryostat windows. The absence of windows greatly simplifies the extraction of the dielectric function. The terahertz transmission in the frequency-domain was obtained by measuring the signal response of quartz and vacuum in the time-domain and dividing the Fourier transformed signal of the quartz by that of vacuum. The method for obtaining the complex refractive index from TDS is described later.

Figure 1a shows the extinction coefficient measured by TDS and FTIR spectroscopy at $10 \mathrm{~K}$ demonstrating the absorption of the first two $E$-symmetry phonon modes of $\alpha$-quartz at $\sim 3.95 \mathrm{THz}\left(132 \mathrm{~cm}^{-1}\right)$ and $\sim 7.94 \mathrm{THz}\left(265 \mathrm{~cm}^{-1}\right)$. Figure $1 \mathrm{~b}$ e shows the refractive index (b and $\mathbf{c}$ ) and extinction coefficient ( $\mathbf{d}$ and $\mathbf{e}$ ) of quartz measured by TDS between 10 and $300 \mathrm{~K}$. Table 1 also tabulates the refractive index of quartz as a function of both frequency and temperature. The lowest energy $E$-mode is measured to have a resonant frequency of $\sim 3.9 \mathrm{THz}\left(132 \mathrm{~cm}^{-1}\right)$ as is demonstrated by the derivative-like feature in the refractive index (Fig. 1c) and peak in the extinction coefficient (Fig. 1e). A more detailed discussion of the $3.9 \mathrm{THz} E$ mode is provided in Section 3.2. If this phonon mode is ignored, the (real) refractive index only has a slight temperature dependence, thus a reasonable phenomenological expression for the refractive index of quartz along its optical axis is,

$$
n(v)=\left\{\begin{array}{ll}
2.0928 & \text { if } 0.5 \mathrm{THz}<v<1 \mathrm{THz} \\
2.0959-0.0066 v+0.0035 v^{2} & \text { if } 1 \mathrm{THz}<v<5.5 \mathrm{THz}
\end{array},\right.
$$

where $\omega=2 \pi v$.

Now we describe the conversion from the terahertz transmission function (with phase information) of the sample to the complex refractive index. Consider an incident propagating monochromatic wave, $E_{\mathrm{i}}=E_{0} e^{i(k z-\omega t)}$ with a dispersion relation $k=\tilde{n} \omega / c$ where $\tilde{n}$ is the complex refractive index of a material. The transmitted electric fields $E_{\mathrm{r}}$ and $E_{\mathrm{s}}$ for (a) vacuum and (b) a thick sample of thickness $d$, respectively (Fig. 2), are defined in the frequency domain by:

$$
\begin{gathered}
E_{\mathrm{r}}=e^{i\left(\tilde{n}_{\mathrm{r}} d\right) \omega / c} E_{\mathrm{i}} \\
E_{\mathrm{s}}=t_{\mathrm{r}, \mathrm{s}} t_{\mathrm{s}, \mathrm{r}} e^{i\left(\tilde{n}_{\mathrm{s}} d\right) \omega / c} E_{\mathrm{i}}
\end{gathered}
$$




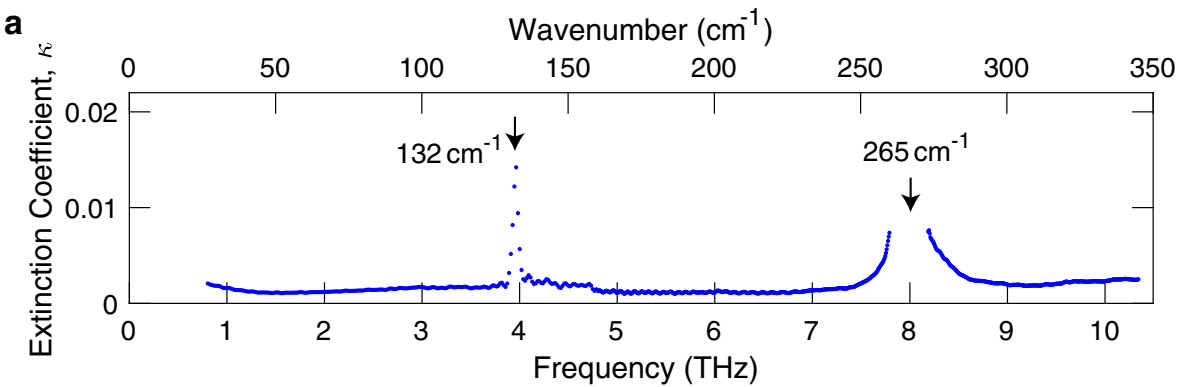

b

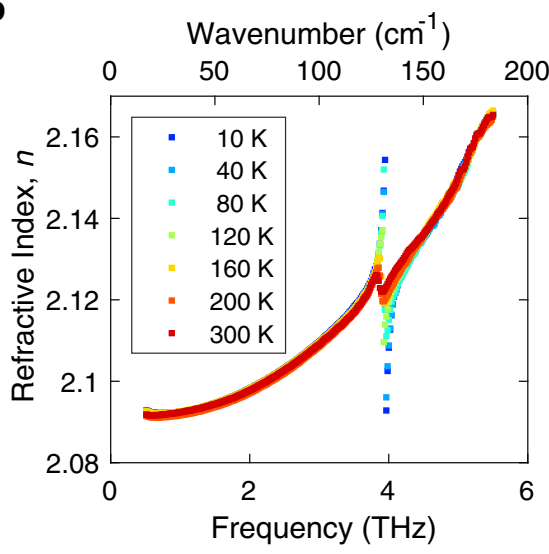

d

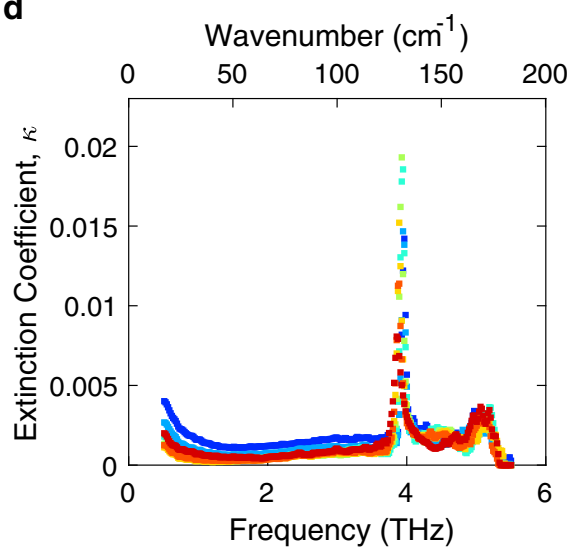

C

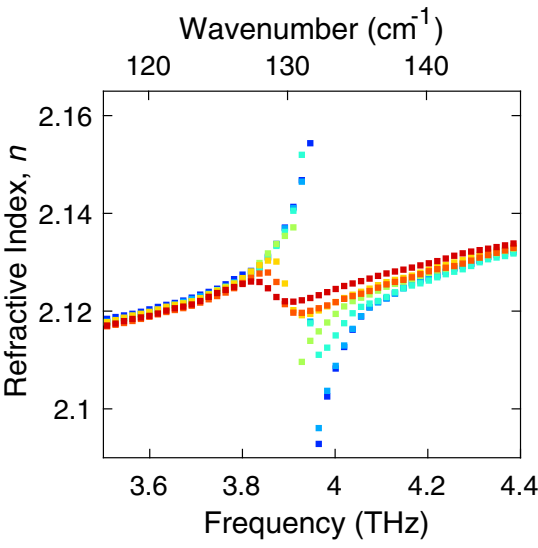

e

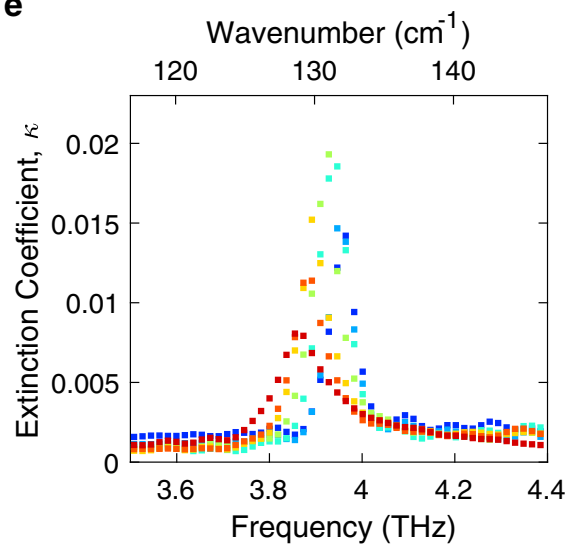

Fig. 1 a Extinction coefficient measured by TDS $(v<4.8 \mathrm{THz})$ and FTIR $(v>4.8 \mathrm{THz})$ spectroscopy at $10 \mathrm{~K}$ showing the absorption of the first two $E$-symmetry phonon modes of $\alpha$-quartz at $3.96 \mathrm{THz}$ $\left(132 \mathrm{~cm}^{-1}\right)$ and $7.94 \mathrm{THz}\left(265 \mathrm{~cm}^{-1}\right)$. b, $\mathbf{c}$ Refractive index and $\mathbf{d}$ and $\mathbf{e}$ extinction coefficient of $\alpha$-quartz as a function of frequency at 10, 40,80,120,160, 200 and $300 \mathrm{~K}$. Refractive indices and extinction coefficients were measured using terahertz time-domain spectroscopy 
Table 1 Refractive index of $\alpha$-quartz

\begin{tabular}{|c|c|c|c|c|c|c|c|c|}
\hline $\begin{array}{l}\text { Frequency } \\
(\mathrm{THz})\end{array}$ & $10 \mathrm{~K}$ & $40 \mathrm{~K}$ & $80 \mathrm{~K}$ & $120 \mathrm{~K}$ & $160 \mathrm{~K}$ & $200 \mathrm{~K}$ & $300 \mathrm{~K}$ & $\begin{array}{l}\text { Wavenumber } \\
\left(\mathrm{cm}^{-1}\right)\end{array}$ \\
\hline 0.512 & 2.093 & 2.092 & 2.092 & 2.093 & 2.093 & 2.092 & 2.092 & 17.1 \\
\hline 0.603 & 2.092 & 2.092 & 2.092 & 2.092 & 2.092 & 2.091 & 2.092 & 20.1 \\
\hline 0.694 & 2.092 & 2.092 & 2.091 & 2.092 & 2.092 & 2.091 & 2.092 & 23.2 \\
\hline 0.786 & 2.092 & 2.092 & 2.092 & 2.092 & 2.092 & 2.091 & 2.092 & 26.2 \\
\hline 0.877 & 2.092 & 2.092 & 2.092 & 2.092 & 2.092 & 2.091 & 2.092 & 29.3 \\
\hline 0.968 & 2.092 & 2.092 & 2.092 & 2.092 & 2.092 & 2.092 & 2.092 & 32.3 \\
\hline 1.06 & 2.093 & 2.092 & 2.092 & 2.092 & 2.093 & 2.092 & 2.093 & 35.3 \\
\hline 1.15 & 2.093 & 2.093 & 2.092 & 2.093 & 2.093 & 2.092 & 2.093 & 38.4 \\
\hline 1.24 & 2.093 & 2.093 & 2.093 & 2.093 & 2.093 & 2.092 & 2.093 & 41.4 \\
\hline 1.33 & 2.094 & 2.093 & 2.093 & 2.093 & 2.094 & 2.093 & 2.094 & 44.5 \\
\hline 1.43 & 2.094 & 2.094 & 2.093 & 2.094 & 2.094 & 2.093 & 2.094 & 47.5 \\
\hline 1.52 & 2.095 & 2.094 & 2.094 & 2.094 & 2.095 & 2.094 & 2.094 & 50.6 \\
\hline 1.61 & 2.095 & 2.095 & 2.095 & 2.095 & 2.095 & 2.094 & 2.095 & 53.6 \\
\hline 1.7 & 2.096 & 2.095 & 2.095 & 2.096 & 2.096 & 2.095 & 2.096 & 56.7 \\
\hline 1.79 & 2.096 & 2.096 & 2.096 & 2.096 & 2.096 & 2.095 & 2.096 & 59.7 \\
\hline 1.88 & 2.097 & 2.097 & 2.096 & 2.097 & 2.097 & 2.096 & 2.097 & 62.8 \\
\hline 1.97 & 2.098 & 2.097 & 2.097 & 2.098 & 2.098 & 2.097 & 2.098 & 65.8 \\
\hline 2.06 & 2.099 & 2.098 & 2.098 & 2.098 & 2.099 & 2.098 & 2.098 & 68.9 \\
\hline 2.16 & 2.1 & 2.099 & 2.099 & 2.099 & 2.1 & 2.099 & 2.099 & 71.9 \\
\hline 2.25 & 2.1 & 2.1 & 2.1 & 2.1 & 2.1 & 2.099 & 2.1 & 75 \\
\hline 2.34 & 2.101 & 2.101 & 2.101 & 2.101 & 2.101 & 2.1 & 2.101 & 78 \\
\hline 2.43 & 2.102 & 2.102 & 2.102 & 2.102 & 2.102 & 2.101 & 2.102 & 81.1 \\
\hline 2.52 & 2.103 & 2.103 & 2.103 & 2.103 & 2.103 & 2.102 & 2.103 & 84.1 \\
\hline 2.61 & 2.104 & 2.104 & 2.104 & 2.104 & 2.104 & 2.103 & 2.104 & 87.1 \\
\hline 2.7 & 2.106 & 2.105 & 2.105 & 2.105 & 2.106 & 2.105 & 2.105 & 90.2 \\
\hline 2.8 & 2.107 & 2.106 & 2.106 & 2.107 & 2.107 & 2.106 & 2.106 & 93.2 \\
\hline 2.89 & 2.108 & 2.108 & 2.107 & 2.108 & 2.108 & 2.107 & 2.108 & 96.3 \\
\hline 2.98 & 2.109 & 2.109 & 2.109 & 2.109 & 2.109 & 2.108 & 2.109 & 99.3 \\
\hline 3.07 & 2.111 & 2.11 & 2.11 & 2.11 & 2.111 & 2.11 & 2.11 & 102 \\
\hline 3.16 & 2.112 & 2.112 & 2.111 & 2.112 & 2.112 & 2.111 & 2.111 & 105 \\
\hline 3.25 & 2.114 & 2.113 & 2.113 & 2.113 & 2.113 & 2.113 & 2.113 & 108 \\
\hline 3.34 & 2.115 & 2.115 & 2.114 & 2.115 & 2.115 & 2.114 & 2.114 & 112 \\
\hline 3.43 & 2.117 & 2.116 & 2.116 & 2.116 & 2.116 & 2.116 & 2.116 & 115 \\
\hline 3.53 & 2.119 & 2.118 & 2.118 & 2.118 & 2.118 & 2.117 & 2.117 & 118 \\
\hline 3.62 & 2.121 & 2.12 & 2.12 & 2.12 & 2.12 & 2.119 & 2.12 & 121 \\
\hline 3.71 & 2.123 & 2.123 & 2.123 & 2.123 & 2.123 & 2.122 & 2.122 & 124 \\
\hline 3.8 & 2.127 & 2.127 & 2.126 & 2.127 & 2.127 & 2.126 & 2.126 & 127 \\
\hline 3.89 & 2.137 & 2.137 & 2.136 & 2.135 & 2.126 & 2.121 & 2.122 & 130 \\
\hline 3.98 & 2.103 & 2.104 & 2.113 & 2.118 & 2.121 & 2.121 & 2.124 & 133 \\
\hline
\end{tabular}


Table 1 (continued)

\begin{tabular}{|c|c|c|c|c|c|c|c|c|}
\hline $\begin{array}{l}\text { Frequency } \\
(\mathrm{THz})\end{array}$ & $10 \mathrm{~K}$ & $40 \mathrm{~K}$ & $80 \mathrm{~K}$ & $120 \mathrm{~K}$ & $160 \mathrm{~K}$ & $200 \mathrm{~K}$ & $300 \mathrm{~K}$ & $\begin{array}{l}\text { Wavenumber } \\
\left(\mathrm{cm}^{-1}\right)\end{array}$ \\
\hline 4.07 & 2.121 & 2.121 & 2.122 & 2.123 & 2.125 & 2.124 & 2.127 & 136 \\
\hline 4.17 & 2.126 & 2.126 & 2.125 & 2.127 & 2.127 & 2.127 & 2.129 & 139 \\
\hline 4.26 & 2.129 & 2.129 & 2.128 & 2.129 & 2.13 & 2.129 & 2.131 & 142 \\
\hline 4.35 & 2.132 & 2.131 & 2.131 & 2.132 & 2.132 & 2.132 & 2.133 & 145 \\
\hline 4.44 & 2.134 & 2.134 & 2.134 & 2.134 & 2.135 & 2.134 & 2.135 & 148 \\
\hline 4.53 & 2.137 & 2.136 & 2.136 & 2.137 & 2.137 & 2.137 & 2.137 & 151 \\
\hline 4.62 & 2.139 & 2.138 & 2.139 & 2.139 & 2.14 & 2.139 & 2.139 & 154 \\
\hline 4.71 & 2.141 & 2.141 & 2.141 & 2.141 & 2.142 & 2.141 & 2.141 & 157 \\
\hline 4.81 & 2.143 & 2.143 & 2.144 & 2.144 & 2.144 & 2.143 & 2.144 & 160 \\
\hline 4.9 & 2.147 & 2.146 & 2.145 & 2.146 & 2.147 & 2.145 & 2.146 & 163 \\
\hline 4.99 & 2.15 & 2.149 & 2.148 & 2.149 & 2.149 & 2.148 & 2.15 & 166 \\
\hline 5.08 & 2.153 & 2.153 & 2.151 & 2.152 & 2.152 & 2.152 & 2.152 & 169 \\
\hline 5.17 & 2.157 & 2.156 & 2.156 & 2.156 & 2.156 & 2.156 & 2.157 & 172 \\
\hline 5.26 & 2.16 & 2.16 & 2.16 & 2.16 & 2.16 & 2.16 & 2.161 & 176 \\
\hline 5.35 & 2.162 & 2.163 & 2.163 & 2.162 & 2.163 & 2.162 & 2.162 & 179 \\
\hline 5.44 & 2.165 & 2.165 & 2.165 & 2.164 & 2.166 & 2.163 & 2.165 & 182 \\
\hline
\end{tabular}

where the subscripts $\mathrm{r}$ and $\mathrm{s}$ denote the vacuum reference and sample, $t_{i, j}=$ $2 \tilde{n}_{i} /\left(\tilde{n}_{i}+\tilde{n}_{j}\right)$ is the Fresnel transmission at the boundary between materials $i$ and $j$. The terahertz sample transmission is given by:

$$
T(\omega)=\frac{E_{\mathrm{s}}}{E_{\mathrm{r}}}=\frac{4 \tilde{n}_{\mathrm{s}}}{\left(1+\tilde{n}_{\mathrm{s}}\right)^{2}} e^{i\left(\left(\tilde{n}_{\mathrm{s}}-1\right) d\right) \omega / c}
$$

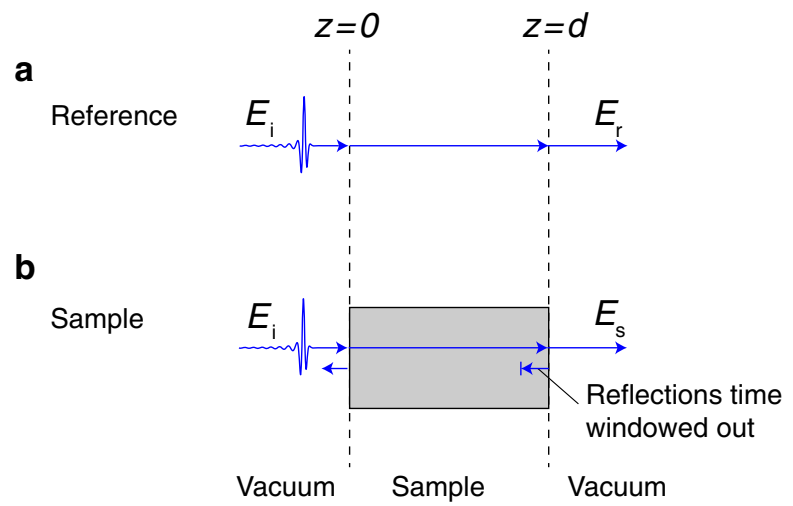

Fig. 2 Transmission of a terahertz pulse through: a vacuum and $\mathbf{b}$ a thick sample of thickness $d$. The reflections from the back surface of the sample are time windowed out during the experiment 
There are no analytic solutions for the complex refractive index, $\tilde{n}_{\mathrm{s}}$, for the above equation so $\tilde{n}_{\mathrm{s}}$ will need to be found numerically. Owing to the complex exponent in Eq. 5, $T(\omega)$ is oscillatory in $n$-space which would lead to convergence issues if a standard least squares fitting method is applied. To overcome this potential fitting difficulty, an alternate error function is used in the fitting algorithm [29, 30], given by:

$$
\xi(n, \kappa)=\xi_{\bmod }^{2}(n, \kappa)+\xi_{\arg }^{2}(n, \kappa)
$$

where $n$ and $\kappa$ are the real and imaginary parts of the complex refractive index. The functions $\xi_{\text {mod }}$ and $\xi_{\text {arg }}$ are defined by:

$$
\begin{gathered}
\xi_{\text {mod }}(\omega ; n, \kappa)=\log (|T(\omega ; n, \kappa)|)-\log \left(\left|T_{\text {meas }}(\omega)\right|\right) \\
\xi_{\text {arg }}(\omega ; n, \kappa)=\arg (T(\omega ; n, \kappa))-\arg \left(T_{\text {meas }}(\omega)\right)
\end{gathered}
$$

where $T_{\text {meas }}$ is the measured value and $T$ is the fitted value. The objective of the fitting algorithm is to minimise the error function, $\xi$.

\subsection{Terahertz Frequency Phonon Mode in $\alpha$-Quartz}

The spectral response of the $3.9 \mathrm{THz} E$-phonon mode in $\alpha$-quartz (Fig. 3) was modelled by a Lorentz oscillator with temperature-dependent fitting parameters. The refractive index was fitted using two Lorentz oscillators to account for the $\sim 3.9 \mathrm{THz}$ $E$-mode and the presence of a second broad $E$-mode at $\sim 8 \mathrm{THz}\left(=265 \mathrm{~cm}^{-1}\right)[12$, 17]. The $A_{1}$ mode at $220 \mathrm{~cm}^{-1}$ is not infrared active and thus does not contribute [12]. The refractive index was fitted with the following function [31],

$$
n(\omega)=\sqrt{\frac{\left|\tilde{\epsilon}_{L}(\omega)\right|+\operatorname{Re}\left\{\tilde{\epsilon}_{L}(\omega)\right\}}{2}}
$$

where $\epsilon_{L}$ is the relative dielectric function of two Lorentz oscillators given by:

$$
\tilde{\epsilon}_{L}(\omega)=\epsilon_{\infty}-\sum_{j=1}^{2} \frac{A_{j} \omega_{j}^{2}}{\omega^{2}-\omega_{j}^{2}-i \Gamma_{j} \omega}
$$

where $\epsilon_{\infty}$ is the high frequency dielectric constant, $A_{j}$ is the oscillator strength, $\omega_{j}$ is the resonant phonon frequency with $\omega_{j=2}$ fixed at $8 \mathrm{THz}$ [12], and $\Gamma_{j}$ is the damping parameter.

Figure 4 shows (a) the oscillator strength, (b) the phonon resonant frequency and (c) the damping parameter as a function of temperature that were extracted by fitting Eq. 9. The damping parameter, $\Gamma$ increases with increasing temperature as expected. Even at $10 \mathrm{~K}$, splitting of the energy degeneracy of the $3.9 \mathrm{THz} \mathrm{LO}$ and TO modes could not be resolved indicating that the splitting is less than $0.8 \mathrm{~cm}^{-1}$, which is in agreement with variational density-functional perturbation theory predictions [32]. The electric dipole oscillator strength decreases with increasing temperature and the phonon resonance redshifts with increasing temperature, in agreement with theoretical predictions based on a model of cubic anharmonicity [18]. This trend is expected 


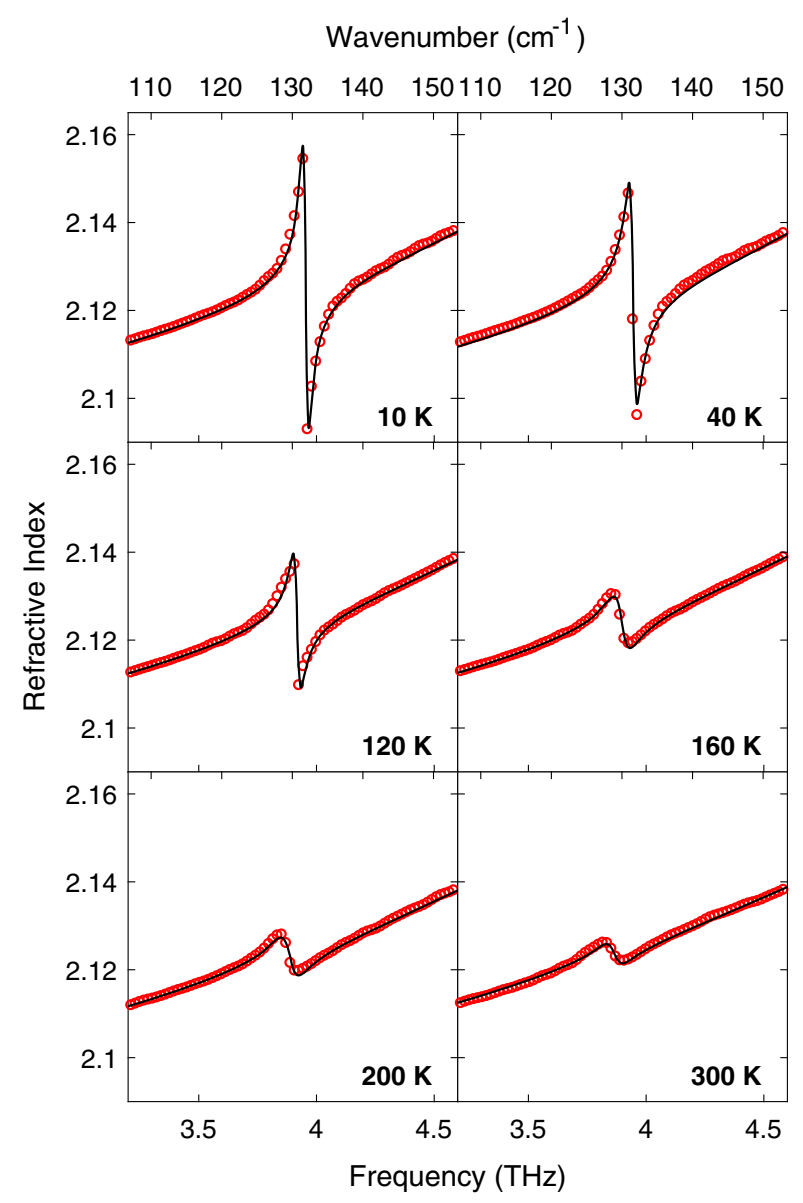

Fig. 3 Refractive index of $\alpha$-quartz near the $3.9 \mathrm{THz}$ phonon mode with the data shown as red circles and fits shown as black lines. The fitted data are modelled by a Lorentz oscillator as described in the text (Eq. 9)

as a harmonic (parabolic) potential is only a good approximation for low vibrational energy levels. At higher temperatures, higher energy vibrational energy levels also become occupied according to a Bose-Einstein distribution and the widening of the potential from a parabola at the higher energies (i.e. the weakening of interatomic forces for large amplitude displacement) means that the energy levels are spaced closer together. Thus, phonon modes generally redshift and broaden at higher temperatures.

\subsection{Polariton Dispersion in $\alpha$-Quartz}

The polariton dispersion relation demonstrates the coupling between a photon and the longitudinal (LO) and transverse (TO) optical phonons. Figure 5 shows the polariton 

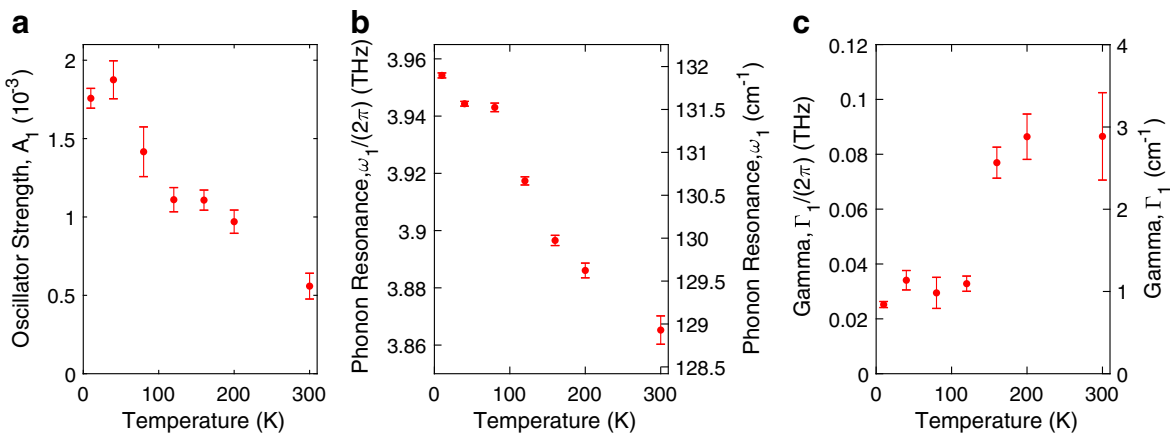

Fig. 4 Extracted parameters from fitting an oscillator model (Eq. 9) to the refractive index near the $3.9 \mathrm{THz}$ phonon mode (Fig. 3) showing a the oscillator strength, $\mathbf{b}$ the phonon resonant frequency, and $\mathbf{c}$ the damping parameter as a function of temperature

dispersion relation of $\alpha$-quartz with the lowest energy $E$ mode near the zone centre. The dispersion relation is a plot of frequency against wave vector, where the wave vector is calculated using the measured refractive index by the following equation [31],

$$
k(\omega)=\frac{\omega|n(\omega)|}{c}
$$

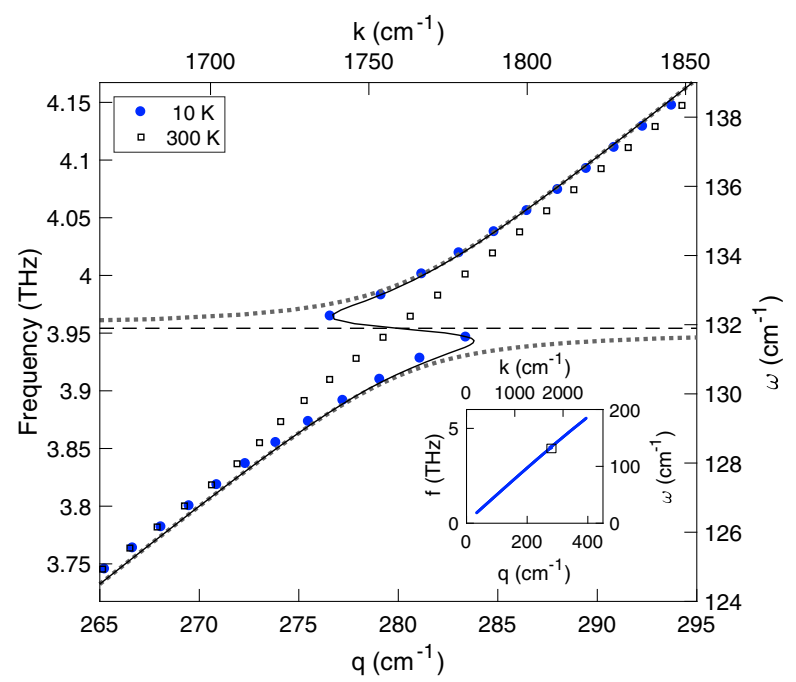

Fig. 5 Dispersion relation of the polariton with the lowest energy doubly degenerate $E$ mode of $\alpha$-quartz measured at $10 \mathrm{~K}$ and $300 \mathrm{~K}$. The inset shows is an enlarged section of the dispersion relation near the $E$ mode. The solid black line is a fit to the dispersion relation at $10 \mathrm{~K}$ using Eq. 12 with the damping parameter set to $\Gamma=5.3 \mathrm{~cm}^{-1}$ from the previous fitting of the refractive index. The dotted grey line is the calculated dispersion relation in the absence of damping, $\Gamma=0 \mathrm{~cm}^{-1}$. The horizontal dashed black line is the energy of the $E$ mode $\left(132 \mathrm{~cm}^{-1} \approx 3.954 \mathrm{THz}\right)$ 
Here, the photon couples with the doubly degenerate $E$ mode and no energy gap between the LO and TO phonons can be resolved. The polariton dispersion relation at $10 \mathrm{~K}$ was fitted with the following equation,

$$
k^{2}=\frac{\omega^{2}}{c^{2}} \epsilon_{\infty}\left(\frac{\omega_{\mathrm{LO}}^{2}-\omega^{2}-i \Gamma \omega}{\omega_{\mathrm{TO}}^{2}-\omega^{2}-i \Gamma \omega}\right),
$$

where $\omega_{\mathrm{LO}}$ and $\omega_{\mathrm{TO}}$ are the frequencies of the LO and TO phonon modes and $\Gamma$ is the damping parameter. $\Gamma$ and average of $\omega_{\mathrm{LO}}$ and $\omega_{\mathrm{TO}}$ were fixed at $5.3 \mathrm{~cm}^{-1}$ and $132 \mathrm{~cm}^{-1}$ respectively, as determined from the previous fitting of the refractive index. $\epsilon_{\infty}$ is the high frequency (i.e. frequencies much greater than $\omega_{\text {LO }}$ ) dielectric constant and was found to be 4.52. The splitting of the LO and TO phonon modes was determined to be less than $0.03 \mathrm{~cm}^{-1}$ and is significantly less than the damping parameter.

\section{Conclusion}

Quartz is a cheap, inert and durable substrate for supporting samples during optical spectroscopy measurements at terahertz frequencies. Here, we have presented the complex refractive index of $\alpha$-quartz at terahertz frequencies $(0.5$ to $5.5 \mathrm{THz})$ at temperatures between 10 and $300 \mathrm{~K}$. $\alpha$-quartz shows excellent transmission properties for spectroscopy over this frequency range apart from a band of $0.2 \mathrm{THz}$ width centred on a phonon absorption feature at 3.9 THz. We have also used terahertz TDS to study the temperature dependence of this phonon feature. It originates from the lowest energy TO and LO phonon modes of quartz, for which we extracted the phonon-polariton dispersion and found the splitting of the degeneracy of the two modes to be less than $0.03 \mathrm{~cm}^{-1}$ at $10 \mathrm{~K}$. The phonon lifetime, energy and dipole oscillator strength were measured as a function of temperature with experimental trends agreeing with previously published theoretical models of anharmonicity. Our results will assist with future optical characterisation of non-free standing samples in the terahertz region, which relies on accurate knowledge of the refractive index and extinction coefficient of the substrate.

Funding information This work was supported by the UK Engineering and Physical Sciences Research Council UK. This project has received funding from the European Union's Horizon 2020 research and innovation programme under grant agreement No 735008.

Open Access This article is distributed under the terms of the Creative Commons Attribution 4.0 International License (http://creativecommons.org/licenses/by/4.0/), which permits unrestricted use, distribution, and reproduction in any medium, provided you give appropriate credit to the original author(s) and the source, provide a link to the Creative Commons license, and indicate if changes were made.

\section{References}

1. Jorge I Cisneros. Optical characterization of dielectric and semiconductor thin films by use of transmission data. Appl. Opt., 37(22):5262-5270, 1998. 
2. Yoichi Murakami, Shohei Chiashi, Yuhei Miyauchi, Minghui Hu, Masaru Ogura, Tatsuya Okubo, and Shigeo Maruyama. Growth of vertically aligned single-walled carbon nanotube films on quartz substrates and their optical anisotropy. Chemical Physics Letters, 385(3):298-303, 2004.

3. Christopher L Davies, Patrick Parkinson, Nian Jiang, Jessica L Boland, Sonia Conesa-Boj, Hark Hoe Tan, C Jagadish, Laura M Herz, and Michael B Johnston. Low ensemble disorder in quantum well tube nanowires. Nanoscale, 7:20531-20538, 2015.

4. Christopher L Davies, Marina R Filip, Jay B Patel, Timothy W Crothers, Carla Verdi, Adam D Wright, Rebecca L Milot, Feliciano Giustino, Michael B Johnston, and Laura M Herz. Bimolecular recombination in methylammonium lead triiodide perovskite is an inverse absorption process. Nat. Commun., 9(1):293, 2018.

5. Patrick Parkinson, James Lloyd-Hughes, Qiang Gao, H Hoe Tan, Chennupati Jagadish, Michael B Johnston, and Laura M Herz. Transient terahertz conductivity of GaAs nanowires. Nano Lett., 7(7):2162-2165, 2007.

6. Rebecca L Milot, Giles E Eperon, Henry J Snaith, Michael B Johnston, and Laura M Herz. Temperature-dependent charge-carrier dynamics in $\mathrm{CH}_{3} \mathrm{NH}_{3} \mathrm{PbI}_{3}$ perovskite thin films. Adv. Funct. Mater., 25(39):6218-6227, 2015.

7. Hannah J Joyce, Jennifer Wong-Leung, Chaw-Keong Yong, Callum J Docherty, Suriati Paiman, Qiang Gao, H Hoe Tan, Chennupati Jagadish, James Lloyd-Hughes, Laura M Herz, and Michael B Johnston. Ultralow surface recombination velocity in InP nanowires probed by terahertz spectroscopy. Nano Lett., 12(10):5325-5330, 2012.

8. Chan La-O-Vorakiat, Huanxin Xia, Jeannette Kadro, Teddy Salim, Daming Zhao, Towfiq Ahmed, Yeng Ming Lam, Jian-Xin Zhu, Rudolph A Marcus, Maria-Elisabeth Michel-Beyerle, and Elbert E M Chia. Phonon mode transformation across the orthohombictetragonal phase transition in a lead iodide perovskite $\mathrm{CH}_{3} \mathrm{NH}_{3} \mathrm{PbI}_{3}$ : A terahertz time-domain spectroscopy approach. J. Phys. Chem. Lett., 7(1):1-6, 2016.

9. Jun Yang, Tianyu Xia, Shuaicheng Jing, Guangsheng Deng, Hongbo Lu, Yong Fang, and Zhiping Yin. Electrically tunable reflective terahertz phase shifter based on liquid crystal. Journal of Infrared, Millimeter, and Terahertz. Waves, 39(5):439-446, may 2018.

10. H J Joyce, J L Boland, C L Davies, S A Baig, and M B Johnston. A review of the electrical properties of semiconductor nanowires: insights gained from terahertz conductivity spectroscopy. Semicond. Sci. Technol., 31:103003, 2016.

11. I H Malitson. Interspecimen comparison of the refractive index of fused silica*,. J. Opt. Soc. Am., 55(10):1205-1209, 1965.

12. F Bréhat and B Wyncke. Measurement of the optical constants of crystal quartz at $10 \mathrm{~K}$ and $300 \mathrm{~K}$ in the far infrared spectral range: $10-600 \mathrm{~cm}^{-1}$. International Journal of Infrared and Millimeter Waves, 18(9):1663-1679, 1997.

13. J Lloyd-Hughes and Tae-In Jeon. A review of the terahertz conductivity of bulk and nano-materials. $J$ Infrared Milli Terahz Waves, 33:871, 2012.

14. K Kihara. An X-ray study of the temperature dependence of the quartz structure. European Journal of Mineralogy, 2(1):63, 1990.

15. E Castro-Camus, J Lloyd-Hughes, L Fu, H H Tan, C Jagadish, and M B Johnston. An ion-implanted InP receiver for polarization resolved terahertz spectroscopy. Opt. Express, 15:7047-7057, 2007.

16. J B Masson and G Gallot. Terahertz achromatic quarter-wave plate. Opt. Lett., 31:265-267, 2006.

17. Xavier Gonze, Douglas C Allan, and Michael P Teter. Dielectric tensor, effective charges, and phonons in $\alpha$-quartz by variational density-functional perturbation theory. Phys. Rev. Lett., 68(24):3603-3606, 1992.

18. A S Pine and P E Tannenwald. Temperature dependence of raman linewidth and shift in alpha-quartz. Phys. Rev., 178:1424-+, 1969.

19. J Etchepar, M Merian, and L Smetanki. Vibrational normal modes of $\mathrm{SiO}_{2} .1$ Alpha and beta quartz. J. Chem. Phys., 60:1873-1876, 1974.

20. C M Randall and R D Rawcliffe. Refractive indices of germanium, silicon, and fused quartz in the far infrared. Applied Optics, 6(11):1889-1895, 1967.

21. L Nordh, G Olofsson, O Appelblad, and L Klynning. The far infrared transmission of crystal quartz at 3K. Infrared Phys., 27:305-308, 1987.

22. B Wyncke, F Bréhat, and H Kharroubi. Spectroscopic studies of optical materials in the far infrared: The case of crystal quartz. International journal of infrared and millimeter waves, 18(2):475-489, 1997. 
23. D Grischkowsky, S Keiding, M van Exter, and C Fattinger. Far-infrared time-domain spectroscopy with terahertz beams of dielectrics and semiconductors. J. Opt. Soc. Am. B-Opt. Phys., 7:2006-2015, 1990.

24. S. S. Dhillon, M. S. Vitiello, E. H. Linfield, A. G. Davies, Matthias C. Hoffmann, John Booske, Claudio Paoloni, M. Gensch, P. Weightman, G. P. Williams, E. Castro-Camus, D. R. S. Cumming, F. Simoens, I. Escorcia-Carranza, J. Grant, Stepan Lucyszyn, Makoto Kuwata-Gonokami, Kuniaki Konishi, Martin Koch, Charles A. Schmuttenmaer, Tyler L. Cocker, Rupert Huber, A. G. Markelz, Z. D. Taylor, Vincent P. Wallace, J. Axel Zeitler, Juraj Sibik, Timothy M. Korter, B. Ellison, S. Rea, P. Goldsmith, Ken B. Cooper, Roger Appleby, D. Pardo, P. G. Huggard, V. Krozer, Haymen Shams, Martyn Fice, Cyril Renaud, Alwyn Seeds, Andreas Stoehr, Mira Naftaly, Nick Ridler, Roland Clarke, John E. Cunningham, and Michael B. Johnston. The 2017 terahertz science and technology roadmap. J. Phys. D-Appl. Phys., 50:043001, 2017.

25. J F Scott and S P S Porto. Longitudinal and transverse optical lattice vibrations in quartz. Phys. Rev., 161(3):903-910, 1967.

26. J L Boland, A Casadei, Tütüncüoglu, F Matteini, C L Davies, F Jabeen, H J Joyce, L M Herz, A Fontcuberta i Morral, and M B Johnston. Increased photoconductivity lifetime in GaAs nanowires by controlled n-type and p-type doping. ACS Nano, 10:4219-4227, 2016.

27. T Kampfrath, M Battiato, P Maldonado, G Eilers, J Nötzold, S Mährlein, V Zbarsky, F Freimuth, Y Mokrousov, S Blügel, M Wolf, I Radu, P M Oppeneer, and M Münzenberg. Terahertz spin current pulses controlled by magnetic heterostructures. Nat. Nanotechnol., 8:256, 2013.

28. T Seifert, S Jaiswal, U Martens, J Hannegan, L Braun, P Maldonado, F Freimuth, A Kronenberg, J Henrizi, I Radu, E Beaurepaire, Y Mokrousov, P M Oppeneer, M Jourdan, G Jakob, D Turchinovich, L M Hayden, M Wolf, M Münzenberg, M Kläui, and T Kampfrath. Efficient metallic spintronic emitters of ultrabroadband terahertz radiation. Nature Photonics, 10:483, 2016.

29. Lionel Duvillaret. A reliable method for extraction of material parameters in terahertz time-domain spectroscopy. IEEE Journal of selected topics in quantum electronics, 2(3):739-746, 1996.

30. Lionel Duvillaret, Frédéric Garet, and Jean-Louis Coutaz. Highly precise determination of optical constants and sample thickness in terahertz time-domain spectroscopy. Applied Optics, 38(2):409, 1999.

31. Mark Fox. Optical properties of solids, 2002.

32. X Gonze, D C Allan, and M P Teter. Dielectric tensor, effective charges, and phonons in alpha-quartz by variational density-functional perturbation-theory. Phys. Rev. Lett., 68:3603-3606, 1992.

33. Ping Zhao et al. Low-temperature-dependent characteristics of Raman scattering in n-type $4 \mathrm{H}-\mathrm{SiC}$. Spectroscopy and Spectral Analysis, 34(1):108-110, 2014. 